\title{
Mathematical Continuity in Dynamic Social Networks
}

\author{
John L. Pfaltz \\ Dept. of Computer Science, University of Virginia \\ jlp@virginia.edu
}

\begin{abstract}
A rigorous concept of continuity for dynamic networks is developed. It is based on closed, rather than open, sets. It is local in nature, in that if the network change is discontinuous it will be so at a single point and the discontinuity will be apparent in that point's immediate neighborhood.

Necessary and sufficient criteria for continuity are provided when the change involves only the addition or deletion of individual nodes or connections (edges).

Finally, we show that an effective network process to reduce large networks to their fundamental cycles is continuous.
\end{abstract}

\section{Introduction}

Networks, or undirected graphs (which we regard as total synonyms) are fundamental for modeling social phenomena [5]. Yet they also abound in both the sciences and humanities, c.f. [17] for its excellent survey and bibliography of over 400 applications. They may be huge; the connectivity of the world wide web is a network - they may be tiny; the atomic bonds in a molecule are an undirected graph.

Such networks are dynamic; yet there has been little formal study of network change [4]. We introduce the concept of network transformation in Section 3. Typically, we are interested in those kinds of transformations which preserve elements of network structure. In particular, we are concerned with "continuous" transformations.

Like open sets in continuous manifolds, closed sets can be a powerful tool for analyzing the structure of discrete systems. Closure is associated with rational choice operators in economics $[12$, $16,15]$. Galois closure can be used to extract rules from data sets for subsequent used in A.I. reasoning systems $[22,23]$. When the system can be partially, or totally, ordered the closed sets are usually intervals, ideals or filters $[11,14]$. In this paper we employ the closed set structure of undirected graphs and networks.

Much of the current mathematical analysis of social networks is statistical $[13,28]$ or combinatoric [27]. Both can provide valuable, broadbrush properties of the entire system. In contrast, our approach focuses on the decomposition of the system into its constituent closed set structure. The closed sets are created by a neighborhood closure introduced in Section 2.1.

In Section 3, we define the concept of continuous transformations of discrete systems in general, and use it in Section 3.1 to explore the behavior of continuous network transformations. All of the mathematical results associated with network closure in these two sections are original. Many of the other results about general closure are not widely available $[20,21,24]$; hence we have provided detailed proofs. These proofs can be skipped without losing the essential content of the paper.

Section 4.1 presents a representative graph reduction process that is applicable to large networks; it is shown to be continuous. Lastly, Section 4.2 introduces the notion of "fuzzy" closure. 


\section{Closure}

An operator $\varphi$ is said to be a closure operator if for all $Y, Z \subseteq P$, it is:

(C1) extensive, $Y \subseteq Y . \varphi$,

(C2) monotone, $Y \subseteq Z$ implies $Y . \varphi \subseteq Z . \varphi$, and,

(C3) idempotent, $Y \cdot \varphi \cdot \varphi=Y \cdot \varphi$.

A subset $Y$ is closed if $Y=Y . \varphi$. In this work we prefer to use suffix notation, in which an operator follows its operand. Consequently, when operators are composed the order of application is read naturally from left to right. With this suffix notation read $Y . \varphi$ as " $Y$ closure". It is well known that the intersection of closed sets must be closed. This latter can be used as the definition of closure, with the operator $\varphi$ defined by $Y . \varphi=\bigcap_{Z_{i} \text { closed }}\left\{Y \subseteq Z_{i}\right\}$.

By a closure system $\mathcal{S}=(P, \varphi)$, we mean a set $P$ of "points" or "elements", together with a closure operator $\varphi$. By $(\mathrm{C} 1)$ the set $P$ must be closed. In a social network these points are typically individuals, or possibly institutions. The empty set, $\varnothing$, may, or may not, be closed.

A point $y \in Y$ is said to be a ordinary point of $Y$ if $y \in(Y-\{y\}) . \varphi$. In contrast, a point $y \in Y$ is said to be an extreme point of $Y$ if $y \notin(Y-\{y\}) . \varphi$. (Extreme points have a central role in antimatroid closure theory $[2,6]$.) A set is said to be whole if all of its points are ordinary points.

\subsection{Neighborhood Closure}

Let $\mathcal{S}=(P, \mathcal{A})$ be a set $P$ of points, or elements, together with a symmetric adjacency relation $\mathcal{A}$. By the neighborhood, or neighbors, of a set $Y$ we mean the set $Y . \eta=\{x \notin Y \mid \exists y \in Y,(x, y) \in \mathcal{A}\}$. By the region dominated by $Y$ we mean $Y . \rho=Y \cup Y . \eta .{ }^{1}$ Suppose $P$ is a set of individuals and the relation $\mathcal{A}$ denotes a symmetric connection, such as mutual communication, between them. The neighborhood $y . \eta$ about a person $y$ is the set of individuals with which $y$ directly communicates. The neighborhood, $Y . \eta$, of a set $Y$ of individuals is the set of individuals not in $Y$ who directly communicate with at least one individual in $Y$. The region, $Y$. $\rho$, also includes $Y$ itself. Members of $Y$ may, or may not, communicate with each other.

We can visualize the neighborhood structure of a discrete set of points, or individuals, as an undirected graph such as Figure 1. The neighbors of any point are those adjacent in the graph. In

\begin{tabular}{|c|c|c|c|c|c|c|c|c|}
\hline & & & & & & & & \\
\hline & & 1 & 1 & 0 & 0 & & & \\
\hline & & 1 & 1 & 1 & 0 & 0 & & \\
\hline & 1 & & & & & & & \\
\hline & 0 & 1 & 1 & 1 & 0 & 0 & & \\
\hline e & 0 & C & 1 & & 1 & 1 & & \\
\hline & 0 & 0 & 1 & 0 & 1 & 1 & & \\
\hline & 0 & 0 & 0 & 1 & 1 & ) & & \\
\hline & & & & & & & & \\
\hline
\end{tabular}

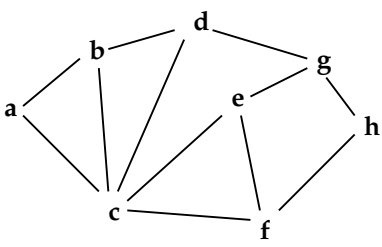

Fig. 1. A symmetric adjacency matrix $\mathcal{A}$ and corresponding undirected graph.

\footnotetext{
${ }^{1}$ In graph theory, $Y . \eta$ is often called the "open neighborhood of $Y$ " and denoted $N(Y)$, while $Y . \rho$, denoted $N[Y]$ has been called the "closed neighborhood of $Y$ " [1,8]. This is a rather different meaning of "closed".
} 
the graph of Figure 1 we have $\{a\} \cdot \eta=\{b, c\}$ or more simply $a . \eta=b c$. And $g . \rho=d e g h$.

Given the neighborhood concepts $\eta$ and $\rho$, we define the neighborhood closure, $\varphi_{\eta}$ to be

$$
Y . \varphi_{\eta}=\{x \mid x . \rho \subseteq Y . \rho\}
$$

In a social system, the closure of a group $Y$ of individuals are those additional individuals, $x$, all of whose connections match those of the group $Y$. A minimal set $X \subseteq Y$ of individuals for which $X . \varphi_{\eta}=Y . \varphi_{\eta}$ is sometimes called the nucleus, core, or generator of $Y . \varphi_{\eta}$. Readily, for all $Y$,

$$
Y \subseteq Y . \varphi_{\eta} \subseteq Y . \rho
$$

that is, $Y$ closure is always contained in the region dominated by $Y$.

Proposition 1. $\varphi_{\eta}$ is a closure operator.

Proof. Readily, $Y \subseteq Y . \varphi_{\eta}$ by definition.

Let $X \subseteq Y$ and let $z \in X . \varphi_{\eta}$. By (1) $z . \rho \subseteq X . \rho \subseteq Y . \rho$ hence $z \in Y . \varphi_{\eta}$.

Let $z \in Y . \varphi_{\eta} . \varphi_{\eta}$. Then $z . \rho \subseteq Y . \varphi_{\eta} . \rho=\bigcup_{x \in Y . \varphi_{\eta}} x . \rho \subseteq Y . \rho$, hence $z \in Y . \varphi_{\eta}$.

Proposition 2. $X . \varphi_{\eta} \subseteq Y . \varphi_{\eta}$ if and only if $X . \rho \subseteq Y . \rho$.

Proof. Let $X . \varphi_{\eta} \subseteq Y . \varphi_{\eta} . \forall x \in X . \varphi_{\eta}, x . \rho \subseteq X . \rho$, so $x \in Y . \varphi_{\eta}$ implies $x . \rho \subseteq Y . \rho$ or $X . \rho \subseteq Y . \rho$. Now suppose $X . \rho \subseteq Y . \rho$. Let $z \in X . \varphi_{\eta}$ implying $z . \rho \subseteq X . \rho \subseteq Y . \rho$ Hence $z \in Y . \varphi_{\eta}$.

An immediate consequence of Proposition 2 is

Corollary 1. $X . \varphi_{\eta}=Y . \varphi_{\eta}$ if and only if $X . \rho=Y . \rho$.

Proposition 3. Let $\varphi_{\eta}$ be the closure operator. If $y . \eta \neq \varnothing$ then there exists $X \subseteq y . \eta$ such that $y \in X . \varphi_{\eta}$.

Proof. Readily, $y . \rho \subseteq y . \eta . \rho$, so $y \in y . \eta . \varphi_{\eta}$. Choose a minimal $X \subseteq y . \eta$ such that $X . \rho \subseteq y . \rho$.

So, unless $y$ is an isolated point, every point $y$ is in the closure of some subset of its neighborhood.

One might expect that every point in a discrete network must be closed, e.g. $\{x\} \cdot \varphi_{\eta}=\{x\}$. But, this need not be true, as shown in Figure 1. The region $c . \rho=a b c d e f$ while $a . \rho=a b c \subseteq c . \rho$ and $b . \rho=a b c d \subseteq c . \rho$, so $c . \varphi_{\eta}=a b c$. The points $a$ and $b$ are ordinary points of $Y=\{a b c\}$, but $Y$ is not whole because $c \notin(a b c-c) \cdot \varphi=\{a b\}$.

Equation (2) suggests an effective computer algorithm to calculate the closure $Y . \varphi_{\eta}$ of any set $Y$. Initially, let $Y . \varphi_{\eta}=Y$; then since $Y . \rho=Y \cup Y . \eta$ examine only the points $z$ in the neighborhood, $Y . \eta$, of $Y$. If $z . \rho \subseteq Y$. $\rho$, add $z$ to $Y . \varphi_{\eta}$.

The following sequence of propositions regarding ordinary points and whole sets all assume that the closure operator is the neighborhood closure. They need not be true in general.

Proposition 4. If $\varphi_{\eta}$ is the closure operator and $y$ is an ordinary point of $Y$, then $y . \rho \subseteq(Y-\{y\}) . \rho \subseteq$

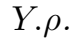


Proof. The first containment follows from the definition of $y \in(Y-\{y\}) \cdot \varphi_{\eta}$. The second containment is always true.

Proposition 5. Let $\varphi_{\eta}$ be the closure operator. If $X$ and $Y$ are finite whole sets and $X \cap Y \neq \varnothing$, then $X=Y$.

Proof. Let $z \in X \cap Y$, so $z$ is an ordinary point of both $X$ and $Y$. By Prop. 4, $z . \rho \subseteq X$. $\rho \cap Y . \rho$. Consequently the iterated neighborhood $z . \rho \ldots \rho \subseteq X \rho \ldots \rho \cap Y \rho \ldots \rho$, and since both are finite this iteration must terminate with $X \subseteq X \cap Y, Y \subseteq X \cap Y$, so $X=Y=X \cap Y$.

It is apparent that with respect to neighborhood closure, whole sets are effectively the non-trivial connected components of the network.

\section{Transformations}

Almost any book on graph theory mentions graph homomorphism, that is a mapping $h:(P, E) \rightarrow$ $\left(P^{\prime}, E^{\prime}\right)$, or a function $h: P \rightarrow P^{\prime}$ in which $(x, y) \in E$ implies that $(h(x), h(y)) \in E^{\prime}[1,8]$. But, a serious limitation of graph homomorphisms is that, since $h: P \rightarrow P^{\prime}$ is a function, the homomorphic "image" must always be "smaller". In the real world, networks expand and contract.

For this reason we introduce the notion of a graph, or network, transformation which is a function mapping the power set, $2^{P}$, of $P$ into the the power set, $2^{P^{\prime}}$, of $P^{\prime}$. That is, every subset of $P$ has a unique image subset in $P^{\prime}$. The operators $\eta, \rho$, and $\varphi_{\eta}$ are transformations of a network $(P, E)$ into itself, since every subset has a unique image. To emphasize this difference, a transformation $f$ is denoted by our suffix notation, e.g. Y.f, rather than the customary prefix notation of functions and homomorphisms. Thus, in neighborhood notation, a graph homomorphism $h$ would be $y . \eta . h \subseteq y . h . \rho^{\prime}$.

We denote transformations of network systems by $(P, E) \stackrel{f}{\longrightarrow}\left(P^{\prime}, E^{\prime}\right)$, or possibly by $(P, \varphi) \stackrel{f}{\longrightarrow}$ $\left(P^{\prime}, \varphi^{\prime}\right)$, since we are often interested in the closure structure induced by the neighborhood system. Note that a transformation $f$ may only change the neighborhood system of $P$ and hence $\varphi^{\prime}$.

In this paper we require that any transformation $f$ be monotone, that is

$$
X \subseteq Y \text { implies } X . f \subseteq Y . f
$$

as seems to be always the case in real applications. Note that "monotone" in this sense only preserves containment relationships; it does not mean that the transformation is "increasing" or "decreasing".

By convention [19,29], a transformation $f$ is said to be continuous if for all $Y \subseteq P$

$$
Y . \varphi . f \subseteq Y . f . \varphi^{\prime}
$$

Readily, (4) holds for all closed sets $Y$ because $Y . \varphi . f=Y . f \subseteq Y . f . \varphi^{\prime}$.

If one visualizes $\varphi$ to be an operative force which causes social cohesion, then "continuity" assures that cohesion observed in the pre-image network will be contained in the cohesion modeled in the resulting image network.

Proposition 6. Let $(P, \varphi) \stackrel{f}{\longrightarrow}\left(P^{\prime}, \varphi^{\prime}\right),\left(P^{\prime}, \varphi^{\prime}\right) \stackrel{g}{\longrightarrow}\left(P^{\prime \prime}, \varphi^{\prime \prime}\right)$ be transformations and let $g$ be monotone. If both $f$ and $g$ are continuous, then so is $P \stackrel{f \cdot g}{\longrightarrow} P^{\prime \prime}$. 
Proof. We have $X . \varphi . f \subseteq X . f . \varphi^{\prime}$ for any $X \in P$ and $Y . \varphi^{\prime} . g \subseteq Y . g . \varphi^{\prime \prime}$ for any $Y \in P^{\prime}$. Consequently, as $g$ is monotone, $X . \varphi . f . g \subseteq X . f . \varphi^{\prime} . g \subseteq X . f . g . \varphi^{\prime \prime}$. Thus $f \cdot g$ is continuous.

Continuous transformations of discrete spaces exhibit many of the properties of continuous real functions with which we are more familiar [26]. For example, let $f$ be a function $f: R \rightarrow R^{\prime}$; if (a) $f$ is onto, then for all $y^{\prime} \in R^{\prime}$ there exists $y \in R$ such that $f(y)=y^{\prime}$; if (b) $f$ is continuous and $X^{\prime}$ is open/closed in $R^{\prime}$, then $f^{-1}\left(X^{\prime}\right)$ is open/closed in $R$; if (c) $f$ is continuous and $X$ is connected in $R$, then $f(X)$ is connected in $R^{\prime}$.

Proposition 7. Let $(P, \varphi) \stackrel{f}{\longrightarrow}\left(P^{\prime}, \varphi^{\prime}\right)$ be monotone, continuous and let $Y^{\prime}=Y$.f be closed. Then $Y . \varphi . f=Y^{\prime}$.

Proof. Let $Y . f$ be closed in $P^{\prime}$. Because $f$ is continuous $Y . \varphi . f \subseteq Y . f . \varphi^{\prime}=Y$.f, since $Y$.f is closed. By monotonicity, $Y . f \subseteq Y . \varphi . f$, so Y. $\varphi . f=Y$.f.

Or, in effect, if the pre-image of a closed set exists it must also be, in a sense, closed.

One can also consider closed transformations which map closed sets in $P$ onto closed sets in $P^{\prime}$. The term "closed transformation" is traditional for structure preserving maps, whether expressed in terms of open sets or closed sets. But, it is most unfortunate in this context, where the multiple meanings can lead to confusion. It is apparent that the composition of closed transformations is another closed transformation.

Proposition 8. A monotone transformation $(P, \varphi) \stackrel{f}{\longrightarrow}\left(P^{\prime}, \varphi^{\prime}\right)$ is closed if and only if $\forall X \subseteq P$, $X . f . \varphi^{\prime} \subseteq X . \varphi . f$.

Proof. Let $f$ be closed. By monotonicity, $X \subseteq X$. $\varphi$ implies $X . f \subseteq X$. $\varphi$.f. But, because $X . \varphi$ is closed and $f$ is closed, $X . f . \varphi^{\prime} \subseteq X . \varphi . f$

Conversely, let all subsets $X \subseteq P$ fulfill $X . f . \varphi^{\prime} \subseteq X . \varphi . f$ and let $X$ be a closed subset of $(P, \varphi)$. Then $X . f . \varphi^{\prime} \subseteq X . f$. But, readily $X . f \subseteq X . f . \varphi^{\prime}$, so equality holds.

Consequently,

Proposition 9. A monotone transformation $(P, \varphi) \stackrel{f}{\longrightarrow}\left(P^{\prime}, \varphi^{\prime}\right)$ is closed and continuous if and only if, for all $X \subseteq P, X . \varphi . f=X . f . \varphi^{\prime}$.

A common way of defining a graph transformation $(P, E) \stackrel{f}{\longrightarrow}\left(P^{\prime}, E^{\prime}\right)$ is to first define $\{y\} . f$ for all singleton sets in $P$ and then extend this to all $Y \subseteq P$ by $Y . f=\bigcup_{y \in Y}\{y\}$. $f$. We call $f$ an extended transformation if $P . f=P^{\prime}$. Any extended transformation is by construction, monotonic.

Proposition 10. If $(P, E) \stackrel{f}{\longrightarrow}\left(P^{\prime}, E^{\prime}\right)$ is an extended transformation, then for all $y^{\prime} \in Y^{\prime}=Y$.f there exists $y \in Y$ such that $y^{\prime} \in\{y\} . f$.

Proof. Let $y^{\prime} \in Y^{\prime}$. By the extended construction $Y^{\prime}=\bigcup_{y \in Y}\{y\} . f$, hence $y^{\prime} \in\{y\} . f$ for some $y \in Y$. 


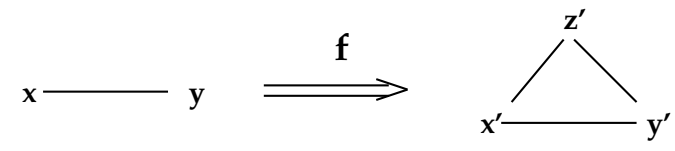

Fig. 2. A simple transformation $f$ with multiple definitions.

Note that this is quite different from asserting a true inverse existence, that for all $y^{\prime} \in Y^{\prime}$, there exists some $y \in Y$ such that $y \cdot f=y^{\prime}$. To get some sense of the import of this "weak inverse existence" proposition, consider the simple transformation $f$ of Figure 2. If we define $f$ on $P$ by $x . f=x^{\prime}$ and $y . f=y^{\prime}$, then by extension $\{x y\} . f=x^{\prime} y^{\prime}$ and $z^{\prime}$ has no pre-image; so $P . f \neq P^{\prime}$. However, if we let $x . f=\left\{x^{\prime} z^{\prime}\right\}, y . f=\left\{y^{\prime} z^{\prime}\right\}$ then $\{x y\} . f=x^{\prime} y^{\prime} z^{\prime}$. Now P.f $=P^{\prime}$, so $f$ is an extended transformation, and Proposition 10 is clearly satisfied.

Unless otherwise explicitly stated, all examples of this paper will be extended transformations.

\subsection{Network Transformations}

The preceding results are true for all closure systems. Now we focus specifically on network transformations. In the next set of propositions it is the neighborhood, $y . \eta$, which is central.

Proposition 11. Let $x \in y . \eta$, then $x \in y \cdot \varphi_{\eta}$ if and only if $x . \rho \subseteq y . \rho$ if and only if $x . \eta-\{y\} \subseteq y . \eta$.

Proof. The first equivalence is simply a restatement of the definition of neighborhood closure. The second equivalence follows because if $x . \rho \subseteq y . \rho$ then $\forall z \neq y, z \in x . \eta$ we have $z \in y . \eta$ and $y \in z . \eta$ by symmetry. The converse is similar.

Proposition 12. Let $(P, E) \stackrel{f}{\longrightarrow}\left(P^{\prime}, E^{\prime}\right)$ be extended. If $f$ is not continuous, there exists $Y \subseteq P$, and $y \in Y . \eta$ such that either

(1) $y^{\prime} \notin Y . f . \eta^{\prime}$

or

(2) $y . \eta \subseteq Y . \eta$ and $y^{\prime} \cdot \eta^{\prime} \nsubseteq \subseteq$ Y.f. $\eta^{\prime}$

Proof. Since $f$ is not continuous, there exists $Y$ such that $Y . \varphi_{\eta} . f \nsubseteq Y . f . \varphi_{\eta}^{\prime}$. Thus, $\exists y^{\prime} \in Y . \varphi_{\eta} . f, y^{\prime} \notin$ $Y . f . \varphi_{\eta}^{\prime}$. By, Prop. 10, $\exists y \in Y . \varphi_{\eta}$ such that $y^{\prime} \in y . f . y \notin Y$ else $y^{\prime} \in Y$.f. Consequently, $y \in Y . \eta$ and $y . \eta \subseteq Y . \eta$. Now, since $y^{\prime} \notin Y . f . \varphi_{\eta}^{\prime}$ we know that either $y^{\prime} \notin Y . f . \eta^{\prime}$ or $y^{\prime} . \eta^{\prime} \not \subset Y . f . \eta^{\prime} . Y$ is technically unspecified, but since $y$ is an ordinary point, by Prop. $11 y \in y . \eta . \varphi_{\eta}$; hence we can assume $\{y\} \subseteq Y \subseteq y \cdot \eta$.

This proposition establishes that if $f$ is discontinuous anywhere, then it will be discontinuous at, or near, a point $y$. One need not consider all subsets of $2^{P}$. Just as is the case with classical function theory, discontinuity, and thus continuity, is a local phenomena. Secondly, it provides conditions (1) and (2) which are are necessary, but not sufficient to demonstrate discontinuity. If for a point $y \in P$ neither condition (1) nor (2) holds, we say $f$ is continuous at $y$. If either condition holds, other criteria must be used, c.f. propositions 13,14 or 16 .

We have said that a transformation $P \stackrel{f}{\longrightarrow} P^{\prime}$ is monotone if $\forall X, Y, X \subseteq Y$ implies $X$. $f \subseteq Y$.f. Let $(P, E) \stackrel{f}{\longrightarrow}\left(P^{\prime}, E^{\prime}\right)$ be a transformation between two neighborhood systems. The transformation 
$f$ is said to be neighborhood monotone if $X . \rho \subseteq Y . \rho$ implies $X . f . \rho^{\prime} \subseteq Y . f . \rho^{\prime}$. A transformation that is monotone need not be neighborhood monotone, and conversely.

Proposition 13. Let $(P, E) \stackrel{f}{\longrightarrow}\left(P^{\prime}, E^{\prime}\right)$ be monotone, then $f$ is continuous if and only if $f$ is neighborhood monotone.

Proof. Let $f$ be continuous and let $X . \rho \subseteq Y . \rho$. By Prop. $2, X \subseteq X . \varphi_{\rho} \subseteq Y . \varphi_{\rho}$. Thus, $X . f \subseteq$ $Y . \varphi_{\rho} . f \subseteq Y . f . \varphi_{\rho}^{\prime}$ by continuity. So X.f. $\rho^{\prime} \subseteq Y . f . \rho^{\prime}$.

Conversely, let $f$ be neighborhood monotone. By definition $Y . \varphi_{\eta}=Y \cup\{x \notin Y \mid x . \rho \subseteq Y . \rho\}$. Since for all $y \in Y, y^{\prime} \in Y$. $f \subseteq Y$.f. $\varphi_{\eta}{ }^{\prime}$, we need only consider $x \notin Y$, but $x . \rho \subseteq Y$. $\rho$. Since $f$ is neighborhood monotone, $x . \rho \subseteq Y . \rho$ implies $x^{\prime} . \rho^{\prime}=x . f . \rho^{\prime} \subseteq Y . f . \rho^{\prime}$ so $x^{\prime} \in Y . f . \varphi_{\eta}{ }^{\prime}$.

Caution: information regarding the region $X$. $\rho$ dominated by a set $X$ reveals very little about $X$ itself. For example, in Figure 1 we have $\{b d\} . \rho=a b c d g \subseteq$ abcdefgh $=\{c g\} . \rho$, yet $\{b d\} \cap\{c g\}=\varnothing$. There is an extensive literature regarding dominating sets, c.f. $[9,10]$.

\subsection{Network Growth}

Unfortunately, both propositions 12 and 13 can be awkward to use in practice. We look for local criteria.

A network can grow by adding points and/or edges. Any transformation which just adds an isolated point $z^{\prime}$ will be continuous, since if $X$ is closed in $(P, \varphi), X^{\prime}$ and $X^{\prime} \cup\left\{z^{\prime}\right\}$ will be closed in $\left(P^{\prime}, \varphi^{\prime}\right)$. But, if continuity is important, care must be taken when adding edges or connections.

Proposition 14. An extended network transformation $f$, which adds an edge $\left(x^{\prime}, z^{\prime}\right)$ to $\mathcal{A}^{\prime}$ at $x$, will be continuous at $x$ if and only if for all $y \in x . \eta, x \in y . \varphi_{\eta}$ implies $z \in y . \eta$.

Proof. First we observe that $x . \varphi_{\eta} . f \subseteq x . f . \varphi_{\eta}^{\prime}$ because $f$ only expands $x^{\prime} . \eta^{\prime}$ so $y \in x . \varphi_{\eta}$ must imply that $y^{\prime} \in x^{\prime} \varphi_{\eta}^{\prime}$.

Moreover, $z \notin x . \eta$ so $\forall y \in x . \eta$ if $w \in y . \varphi_{\eta}, w \neq x$, then $w^{\prime} \in y . \varphi_{\eta} . f$ or $w^{\prime} \in y^{\prime} . \varphi_{\eta}^{\prime}$ because the neighborhoods of $y$ and $w$ are unchanged.

However, $x \in y . \varphi_{\eta}$ implies $x . \rho \subseteq y . \rho$, hence by Prop. $13, f$ is continuous iff $x . \rho^{\prime} \subseteq y . \rho^{\prime}$ iff $z^{\prime} \in y . \rho$.

The transformation $f_{1}$ in Figure 3 which adds the two edges $\left(d^{\prime}, i^{\prime}\right)$ and $\left(g^{\prime}, i^{\prime}\right)$ to $G_{1}$ satisfies Prop. 14. For example, $d \notin b . \varphi_{\eta}=a b, d \notin c . \varphi_{\eta}=a b c$ and $d \notin g \cdot \varphi_{\eta}=g$, so the proposition is trivially satisfied. Similarly, examination at $g$ shows that for all $y \in g . \eta, y=y \cdot \varphi_{\eta}$, so $f_{1}$ is continuous at $g$ as well. Elsewhere it is the identity map so $f_{1}$ is continuous everywhere. We observe that $f_{1}$ is not a closed transformation because $\{d g\}$ is closed in $G_{1}$, but $\left\{d^{\prime} g^{\prime}\right\}$ is not closed in $G_{2}$ because $\{d g\} \cdot \varphi_{\eta}=d^{\prime} g^{\prime} i^{\prime}$.

Expansion of $G_{2}$ at $a^{\prime}$ by creating the edge $\left(a^{\prime \prime}, j^{\prime \prime}\right)$ is different. Because $a^{\prime} \in b^{\prime} . \varphi_{\eta}^{\prime}$ (and $c^{\prime} . \varphi_{\eta}^{\prime}$ ), but $\left(b^{\prime}, j^{\prime}\right) \notin \mathcal{A}^{\prime}$, by Prop. $14 f_{2}$ is discontinuous at $b$ (and also $c$ ). We would also observe that $f_{2}$ is not neighborhood monotone at $b^{\prime}$ because $a^{\prime} \cdot \eta^{\prime}=a^{\prime} b^{\prime} c^{\prime} \subseteq b^{\prime} \cdot \eta^{\prime}=a^{\prime} b^{\prime} c^{\prime} d^{\prime}$ but $a^{\prime \prime} . \eta^{\prime \prime}=a^{\prime \prime} b^{\prime \prime} c^{\prime \prime} j^{\prime \prime} \nsubseteq$ $b^{\prime \prime} \cdot \eta^{\prime \prime}=a^{\prime \prime} b^{\prime \prime} c^{\prime \prime} d^{\prime \prime}$, so $f_{2}$ is not continuous by Prop. 13 as well. Finally, we verify that $b^{\prime} \cdot \varphi_{\eta}^{\prime} \cdot f_{2}=$ $a^{\prime \prime} b^{\prime \prime} \nsubseteq b^{\prime \prime}=b^{\prime \prime} . \varphi_{\eta}^{\prime \prime}$. As this example illustrates, the discontinuity need not occur at either $x$ or $z$, but often at some point $y$ in $x . \eta$ or $z . \eta$ 

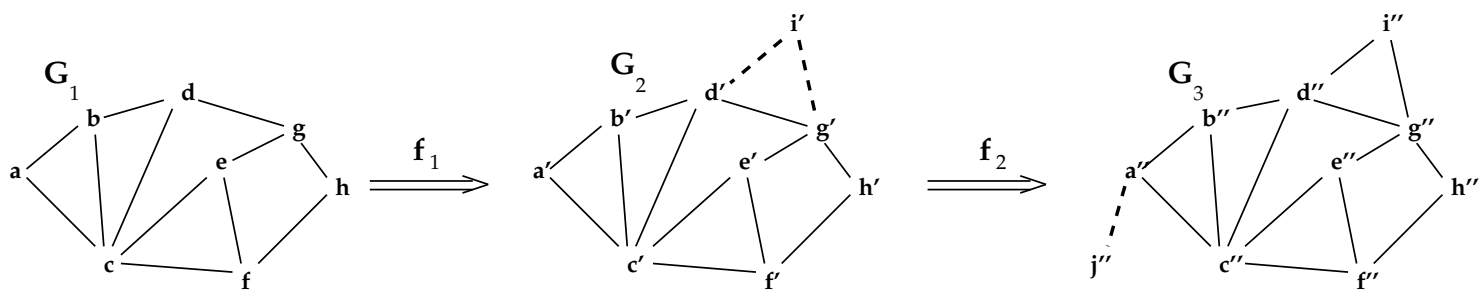

Fig. 3. Two network transformations, $f_{1}$ and $f_{2}$.

\subsection{Network Contraction}

Real networks lose members and connections; but this is hard to model mathematically with homomorphic functions. The problem is that every point in the existing network must map to some point in the image space - and to be a homomorphism it must bring its edges/connections with it. Of course, if the two network elements are truly combined in reality then homomorphism is the right model. But, when the member or connection simply disappears, it isn't.

When we use the transformation model of this paper we can map a point, or subset, onto the empty set, $\varnothing$. We call it point removal. Removal of any point, or node $z$, must also delete all edges incident to $z$, that is all edges of the form $(y, z) \in E$. This is equivalent to deleting a row and column from the adjacency relation, $\mathcal{A}^{\prime}$. We let $\delta_{z}$ denote the removal of $z$ from $P^{\prime}$ and $(y, z)$ from $E^{\prime}$ for all $y \in z . \eta$.

Proposition 15. $\delta_{z}$ is continuous at all $y \in z . \eta$.

Proof. Let $X . \rho \subseteq Y . \rho$. Readily, $X . \rho-\{z\} \subseteq Y . \rho-\{z\}$, so $X . \rho . \delta_{z} \subseteq Y . \rho . \delta_{z}$ and by Prop. $13 \delta_{z}$ is continuous.

Instead of deleting a point and all its incident edges we can remove one, or more, connections by changing the neighborhood structure represented by $\mathcal{A}^{\prime}$.

Proposition 16. An extended network transformation $f$, which deletes an edge $\left(x^{\prime}, z^{\prime}\right)$ from $\mathcal{A}^{\prime}$ at $x$, will be continuous at $x$ if and only if either $z \notin x \cdot \varphi_{\eta}$ or $x \cdot \varphi_{\eta}=z \cdot \varphi_{\eta}$.

Proof. If $z \in x . \varphi_{\eta}$ and $x . \varphi_{\eta} \neq z . \varphi_{\eta}$, then $f$ must be discontinuous because $z^{\prime} \notin x^{\prime} . \eta^{\prime}$ so $x . \varphi_{\eta} . f \nsubseteq$ $x . f \cdot \varphi_{\eta}^{\prime}$.

Now, consider $y \in x . \eta, y \neq z$ so $x \in y . \eta$ by symmetry. If $x \in y . \varphi_{\eta}$ then $x . \eta \subseteq y . \eta$. Since $\mathcal{A}^{\prime}=$ $\mathcal{A}-\left(x^{\prime}, z^{\prime}\right), x^{\prime} \cdot \eta^{\prime} \subseteq x . f . \eta$ or $y \cdot \varphi_{\eta} . f \subseteq y \cdot f . \varphi_{\eta}^{\prime}$.

The second condition, $x \cdot \varphi_{\eta}=z \cdot \varphi_{\eta}$, is needed only for situations such as that of Figure 4 in which $x \cdot \varphi_{\eta}=z \cdot \varphi_{\eta}$ regardless of what other nodes are connected to $y_{1}$ and $y_{2}$. Addition, or deletion, of the dashed edge $(x, z)$ makes no change in the closed set structure whatever.

The individual deletions $\delta_{g^{\prime}}$ and $\delta_{h^{\prime}}$ in Figure 5, The transformations $f_{3}$ and $f_{4}$ of Figure 5 illustrate network contractions. In Figure 5, the dashed edges of $G_{i}$ indicate the deletions in $G_{i+1}$.

By Prop. 16, removing the edge $(a, b)$ from $G_{3}$ is discontinuous. Indeed, we find that $a . \varphi_{\eta} \cdot f_{3}=$ $a^{\prime} b^{\prime} \nsubseteq a . f . \varphi_{\eta}^{\prime}=a^{\prime}$. However, $f_{3}$ is continuous at $c \in a . \eta$. The transformation $f_{4}$ illustrates that rather large network changes can be continuous, since by Proposition 15 both $\delta_{g^{\prime}}$ and $\delta_{h^{\prime}}$ are continuous, 


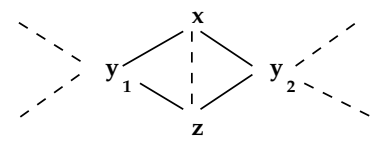

Fig. 4. Two points where $x \cdot \varphi_{\eta}=z \cdot \varphi_{\eta}$.
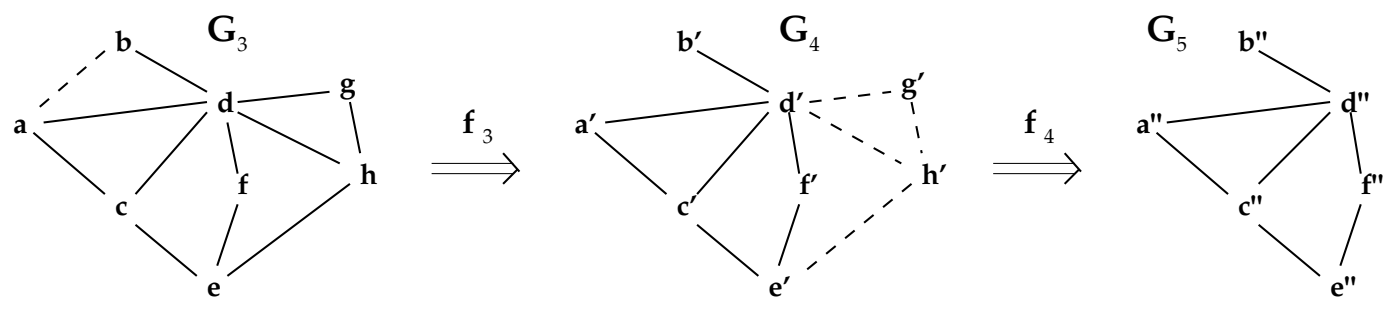

Fig. 5. Contraction of a network by two successive deletions.

and by Proposition $6, G_{4} . \delta_{g^{\prime}} . \delta_{h^{\prime}}$ must be continuous as well. However, removal of either connection $\left(d^{\prime}, g^{\prime}\right)$ or $\left(g^{\prime}, h^{\prime}\right)$ individually would be discontinuous. By Prop. 6 the composition of continuous transformations must be continuous; but as $f_{4}$ illustrates, a continuous transformation need not be decomposable into primitive continuous steps.

In Propositions 14 and 16 we established continuity criteria for network transformations which added and/or deleted elements or connections in a network. But, transformations can radically alter the adjacency structure as shown in Figure 6 and still be continuous. Here, the graph $G_{7}$ is the continuous image of $G_{6}$ under $f_{6}$. This is true because the only neighborhoods of $G_{6}$ are $a b c, a b d$,

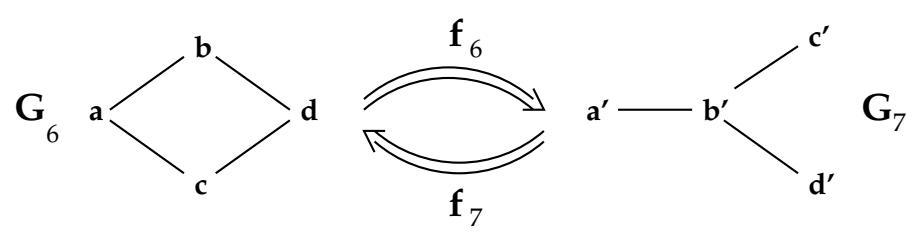

Fig. 6. $f_{6}$ is continuous, $f_{7}$ is not.

$a c d, b c d$ and $a b c d$ so Proposition 13 is trivially satisfied. On the other hand, $c^{\prime} . \rho=b^{\prime} c^{\prime} \subseteq a^{\prime} b^{\prime} c^{\prime} d^{\prime}=$ $b^{\prime} . \rho$, but $c^{\prime} . f_{7} \cdot \rho=a^{\prime} c^{\prime} d^{\prime} \nsubseteq a^{\prime} b^{\prime} d^{\prime}=b^{\prime} . f_{7} \cdot \rho$. So $f_{7}$ cannot be continuous.

\section{Continuity in Practice}

\subsection{Network Reduction}

In Figure 1 of Section 2.1, we observed that the point $c$ is not closed, that $a$ and $b$ are elements of $c . \varphi_{\eta}$. Although $\{a\}$ and $\{b\}$ are themselves closed sets, they must be contained in any closed set containing $c$. We say a point $z$ is subsumed by a set $Y$ if $z$ is an ordinary point of $Y$, that is (by Prop. 4) if $z . \rho \subseteq Y . \rho$. For the reduction process we describe below we will only consider singleton 
sets $Y$, such as $\{c\}$. In a sense, subsumed points such as $a$ and $b$ of Figure 1 contribute little to the closure structure, or topology, of the network. They can be eliminated with little loss of structural information.

In [25], Richards and Seay provide a small 18 point network called the "Sampson" data. They use it to contrast various eigenvector algorithms; we will use it to illustrate graph reduction by point subsumption. Figure 7(a) is one visualization of this network. The circled points of Figure

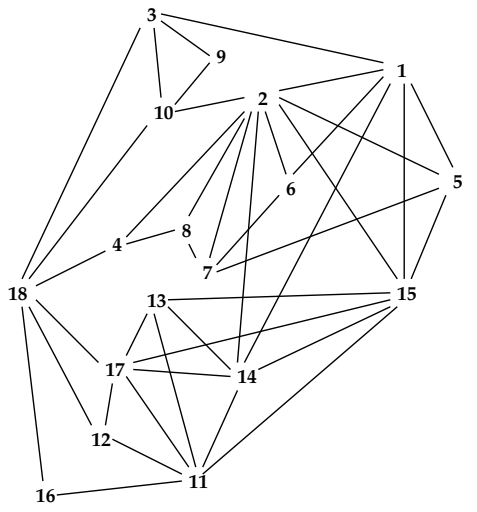

(a)

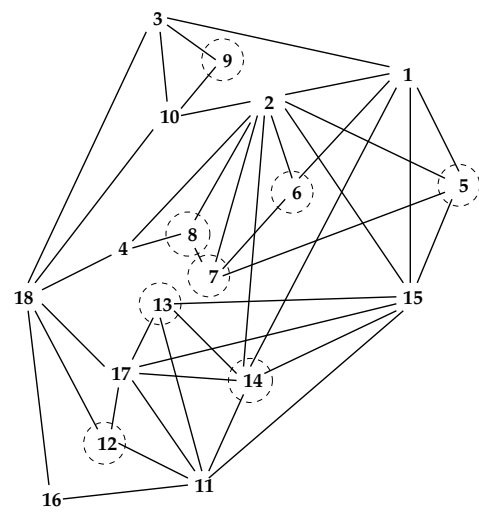

(b)

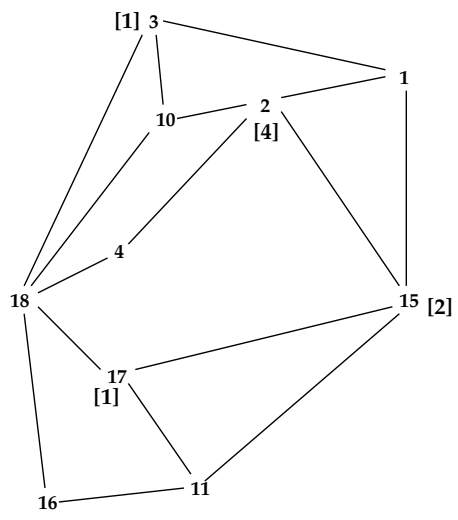

(c)

Fig. 7. (a) Original "Sampson" network, (b) subsumed points, (c) reduced network.

7(b) denote all the points that are subsumed by other singleton sets. For example, 7 is subsumed by 2,14 is subsumed by 15 . Finally, Figure 7 (c) is the reduced graph created by deleting all subsumed points.

The reduced graph of Figure 7(c) is structurally simpler, yet its topology is faithful the the original. By recording [in brackets] the number of points subsumed by each individual node it also conveys a measure of the original density near that node. The key elements of Figure $7(\mathrm{c})$ are chordless cycles of length 4 or greater. These are $\langle 3,10,2,1,3\rangle,\langle 18,4,2,15,17,18\rangle$ and $<18,17,11,16,18>$ in the figure. These are fundamental cycles; no point on a fundamental cycles can be subsumed by another. These fundamental cycles define the topology of the network in much the same manner that 1-cycles can be used to define the topological structure of manifolds [7]. By Proposition 15 the removal of subsumed points, such as $\delta_{7}$ in Figure 7(b) above, are each individually continuous. Thus by Proposition 6 , their composition is continuous.

Figure 7(a) is rather simple to begin with. The continuous reduction by subsumed points is more useful in larger, more complex networks. In [18], Newman presents a 379 node network of collaborating scientists in which each edge denotes at least one co-authored paper. This was reduced by the same program that generated Figure 7 (c) to the 65 node network shown in Figure 8 . As in Figure $7(\mathrm{c})$, values $[n]$ in brackets denote the number of nodes directly, or indirectly, subsumed by the retained node. Dashed lines crudely approximate the extent of nodes in the original network. All of the retained nodes lie on at least one fundamental cycle.

The reduced representation in terms of fundamental cycles is shown in Figure 8. It is a continuous image of the original 379 node network. 


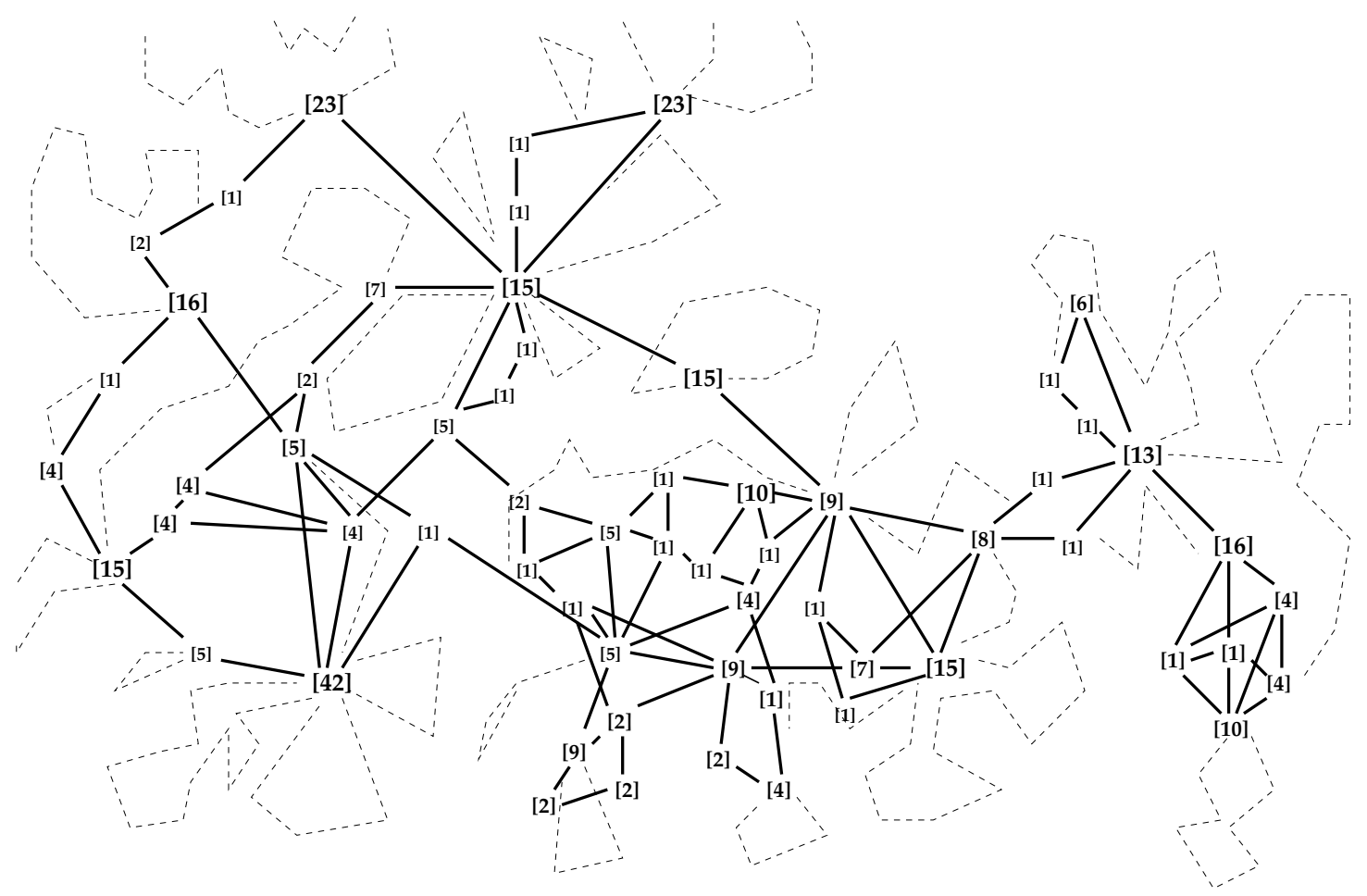

Fig. 8. Fundamental cycles in the collaboration network of [18].

\subsection{Fuzzy Closure}

With neighborhood closure, as defined in Section 2.1, a point $z$ in the neighborhood of a set $Y$ is in $Y$-closure if its neighborhood, $z . \eta$ is completely contained in $Y . \rho$. Thus for $z$ to be subsumed by a single point $y$, as in Section 4.1, all the neighbors/connections of $z$ must already be neighbors of $y$. This is asking for a great deal, and it is rather surprising that the form of network reduction described above works as well is it does on real networks.

When $y$ and $z$ are individuals we would be more likely to say $z$ is tightly bound to $y$ if "almost all" of $z$ 's attachments/connections/neighbors are neighbors of $y$. Can such a fuzzy concept of closure be made rigorous?

Let us define a fuzzy neighborhood closure, $\varphi_{f}$ by $Y . \varphi_{f}=Y \cup\{w \in Y . \eta:|w . \rho-Y . \rho| \leq 1\}$, that is $w$ can have one independent attached neighbor and still be considered to be in the closure $Y . \varphi_{f}$. We use the intersection property of closure systems to show:

Proposition 17. $\varphi_{f}$ is a closure operator.

Proof. Let $X$ and $Z$ be closed w.r.t. $\varphi_{f}$. We claim that $Y=X \cap Z$ is also closed w.r.t. $\varphi_{f}$, that is $Y . \varphi_{f}=Y$. Suppose not, then $\exists w \in(X \cap Z) . \varphi_{f}, w \notin X \cap z$. Let $y \in(X \cap Z) . \varphi_{f}$. If $y \notin X$, there exist at least two neighbors $u, v \in y . \eta, u, v \notin X$, so $u, v \notin X \cap Z$ contradicting the assumption that 
$y \in(X \cap Z) \cdot \varphi_{f}$. So $y \in X$.

Assuming $y \notin Z$ leads to precisely the same contradiction, so $y \in X \cap Z$.

Readily, $Y \subseteq Y . \varphi_{\eta} \subseteq Y . \varphi_{f}$ so this fuzzy closure yields a coarser network structure. For example, the only non-trivial fuzzy closed sets of the graph of Figure 1 are $a b d$, efgh, and $h$

Because $\varphi_{f}$ is a closure operator, many of the preceding propositions are still valid; some are not. For example, the fundamental property (2) does not hold; $Y . \varphi_{f} \not \subset Y . \rho$. If $\mathcal{S}=(Z, \mathcal{A})$ with $Z$ being the integers $\{1, \ldots, n\}$ and $(i, i+1) \in \mathcal{A}$, then the only closed sets are $\varnothing$ and $Z$. No non-empty subset of $Z$ can be closed. Because of the behavior of fuzzy closure in this last example, reduction of the network of Figure 8 using it yields only a single point! Nevertheless, the fact that one can define a fuzzy closure indicates the possibility of use in other kinds of social network analysis.

\section{Summary}

The results of this paper provide a rigorous mathematical foundation for studying the continuous transformation of large social networks. The characterization is based on local changes to the graph, or network, not massive, global transformations. But, "continuity" has always been a local concept couched in terms of very small changes in the pre-image space. ${ }^{2}$ However, Proposition 6 , the example of $f_{4}$ in Figure 5, and our application of Proposition 15 to network reduction demonstrate that global change, which is the composition of smaller continuous steps, may also be characterized as "continuous".

Unlike the traditional approach to continuity, the concept of the "closed set" structure of a network is fundamental. Perhaps the idea of a neighborhood, Y. $\eta$, comes closest to embodying the concept of "nearby points", and thus an "open" set. ${ }^{3}$ However, neighborhoods have few of the key properties of open sets, and trying to fit them into this role seems futile.

Mathematics is a formal exercise; yet surprisingly often it seems to mirror reality. For example, if connections are between individuals, as in social networks, then Proposition 14 would say that creating a connection $(x, z)$ between two persons, $x$ and $z$ where $x$ is closely bound to a third individual $y$, is smoother, easier, or continuous if a connection already exists between $y$ and $z$. This seems to be the case in numerous studies cited by [5].

On the other hand, Proposition 16 would assert that breaking a connection between $x$ and $z$ represents a discontinuity if $z$ is tightly bound to $x$, that is has the same shared connections to others nearby. This also seems to be true in the real world.

While, the introduction of closed sets to the study of transformational change has resolved a number of key issues, there are many more yet to explore. For example, suppose there exists a bicontinuous transformation $f$ between two graphs $G$ and $G^{\prime}$. In what way would they be similar? We might observe that we have yet to encounter a bi-continuous transformation other than a plain isomorphism: it may be that none can exist. In Section 4.2, we show that a form of fuzzy closure can be defined, but we have not explored it rigorously. We only know that our reduction program, using fuzzy closure, always results in a network with only a single node! But, what properties might fuzzy continuity have?

\footnotetext{
${ }^{2}$ E.g. the typical $\epsilon-\delta$ definition of real analysis [26].

${ }^{3}$ Many graph theory texts say that $Y . \eta$ is an "open" neighborhood, c.f. $[1,3,8]$.
} 
Similarly, we have assumed that the relation $\mathcal{A}$ is symmetric. But, many relationships, including friendship, need not be reciprocal. Is neighborhood closure well-defined for non-symmetric relations? Only Proposition 16 explicitly assumes symmetry; but it may be implicitly necessary elsewhere.

Even with all these questions, we believe we have shown that a mathematically rigorous analysis of large social networks based on closed sets can be quite rewarding.

\section{References}

1. Geir Agnarsson and Raymond Greenlaw. Graph Theory: Modeling, Applications and Algorithms. Prentice Hall, Upper Saddle River, NJ, 2007.

2. Kazutoshi Ando. Extreme point axioms for closure spaces. Discrete Mathematics, 306:3181-3188, 2006.

3. Mehdi Behzad and Gary Chartrand. Introduction to the Theory of Graphs. Allyn and Bacon, Boston, 1971.

4. Romain Bourqui, Frederic Gilbert, Paolo Simonetto, Faraz Zaidi, Umang Sharan, and Fabien Jourdan. Detecting structural changes and command hierarchies in dynamic social networks. In 2009 Advances in Social Network Analysis and Mining, pages 83-88, Athens, Greece, 2009.

5. Nicholas A. Christakis and James H. Fowler. Connected, The surprising Power of Our Social Networks and How They Shape Our Lives. Little Brown \& Co., New York, 2009.

6. Paul H. Edelman and Robert E. Jamison. The Theory of Convex Geometries. Geometriae Dedicata, 19(3):247-270, Dec. 1985.

7. P. J. Giblin. Graphs, Surfaces and Homology. Chapman and Hall, London, 1977.

8. Frank Harary. Graph Theory. Addison-Wesley, 1969.

9. Teresa W. Haynes, Stephen T. Hedetniemi, and Peter J. Slater, editors. Domination in Graphs, Advanced Topics. Marcel Dekker, New York, 1998.

10. Teresa W. Haynes, Stephen T. Hedetniemi, and Peter J. Slater. Fundamentals of Domination in Graphs. Marcel Dekker, New York, 1998.

11. Dragan Jankovic and T.R. Hamlett. New Topologies from Old via Ideals. Amer. Math. Monthly, 97(4):295-310, Apr. 1990.

12. G. A. Koshevoy. Choice functions and abstract convex geometries. Mathematical Social Sciences, 38(1):35-44, 1999.

13. Jure Leskovec, Kevin J. Lang, Anirban Dasgupta, and Michael W. Mahoney. Statistical Properties of Community structure in Large Social and Information Networks. In WWW 2008, Proc. of 17th International Conf. on the World Wide Web, pages 695-704, 2008.

14. Terry A. McKee and Fred R. McMorris. Topics in Intersection Graph Theory. SIAM Monographs on Discrete Mathematics and Applications. Society for Industrial and Applied Math., Philadelphia, PA, 1999.

15. Bernard Monjardet. Closure operators and choice operators: a survey. In Fifth Intern. Conf. on Concept Lattices and their Applications, Montpellier, France, Oct. 2007. Lecture notes.

16. Bernard Monjardet and V. Raderinirina. The duality between the antiexchange closure operators and the path independent choice operators on a finite set. Math. Social Sciences, 41(2):131-150, 2001.

17. Mark E. J. Newman. The structure and function of complex networks. SIAM Review, 45:167-256, 2003.

18. Mark. E. J. Newman. Finding community structure in networks using the eigenvectors of matrices. Phys.Rev.E, 74(036104):1-22, July 2006.

19. Oystein Ore. Mappings of Closure Relations. Annals of Math., 47(1):56-72, Jan. 1946.

20. John L. Pfaltz. Closure Lattices. Discrete Mathematics, 154:217-236, 1996.

21. John L. Pfaltz. A Category of Discrete Partially Ordered Sets. In Geir Agnarsson and Jay Shapiro, editors, Mid-Atlantic Algebra Conf., George Mason Univ., Fairfax VA, Nov. 2004.

22. John L. Pfaltz. Logical Implication and Causal Dependency. In Henrik Schärfe, Pascal Hitzler, and Peter Øhrstrøm, editors, Conceptual Structures: Inspiration and Application, volume Springer Verlag LNAI 4068 (supplemental volume), pages 145-157, Aalborg University, July 2006. 
23. John L. Pfaltz. Establishing Logical Rules from Empirical Data. Intern. Journal on Artificial Intelligence Tools, 17(5):985-1001, Oct. 2008.

24. John L. Pfaltz and Josef Šlapal. Neighborhood Transformations. In 40th Southeastern International Conf. on Combinatorics, Graph Theory and Computing, Boca Raton, FL, March 2009.

25. William Richards and Andrew Seary. Eigen Analysis of Networks. J. of Social Structure, 1(2):1-16, 2000.

26. H. L. Royden. Real Analysis. Mcmillian, New York, 1988.

27. A. Saito, editor. Graphs and Combinatorics. Springer, 2010. ISSN 0911-0119.

28. Padhraic Smyth. Statistical Modeling of Graph and Network Data. In Proc. IJCAI Workshop on Learning Statistical Models from Relational Data, Acapulco, Mexico, Aug. 2003.

29. Josef Šlapal. A Galois Correspondence for Digital Topology . In K. Denecke, M. Erné, and S. L. Wismath, editors, Galois Connections and Applications, pages 413-424. Kluwer Academic, Dordrecht, 2004. 